Pecvnia, 8 (2009), pp. 121-147

\title{
La participación de los trabajadores en materia de Prevención de Riesgos Laborales
}

\author{
María Purificación García Miguélez \\ mpgarm@unileon.es \\ Universidad de León \\ Fac. de Ciencias del Trabajo \\ Campus de Vegazana, $\mathrm{s} / \mathrm{n}$ \\ 24071 León (España)
}

El derecho de participación reconocido a los trabajadores para la organización y planificación de las actividades de prevención en las empresas entraña una estructura compleja en diferentes ámbitos.

En primer lugar, respecto al dominio privado e interno, integrado por una participación directa e individualizada de los trabajadores en entidades de plantillas reducidas, o un procedimiento indirecto, colectivo o representativo, en el caso de empresas con un número suficiente de trabajadores para elegir representantes (tanto a través de una representación general -unitaria o sindical- como de una especializada -delegados de prevención y comité de seguridad y salud-).

Son analizados tanto el aspecto "orgánico" (esto es, los órganos representativos precisos para un correcto ejercicio) como el "funcional" (es decir,
The right that is recognized to workers in order to participate in the organization and planning of prevention activities in enterprises entails a complex structure related to different scopes.

Firstly, related to the internal and private domain, consisting in a direct and individualized participation of workers in those entities of small size in staff, or an indirect, collective or representative procedure, in case of enterprises with a number of workers enough to elect representatives (either a general delegation -unit and trade union- or a specialized one -prevention risks delegates and committee on security and health-).

The "organic" aspect (i.e. representative organs required to a proper practice) and the "functional" one (i.e. faculties and competences 
las facultades y competencias a desempeñar), así como las diferencias para ejercer los derechos de información y de consulta, todo ello a fin determinar el órgano de representación más idóneo en cada caso

En segundo lugar, y en relación con el marco de actuación público, el derecho puede ser denominado de participación representativa e institucional, pues es llevada a cabo en diferentes órganos administrativos y fundaciones sectoriales, siendo los sindicatos más representativos responsables de su correcto ejercicio.

Palabras clave: Prevención de riesgos laborales; derecho de participación; derecho de información y consulta; representación general y específica. to be performed) are both under analysis. Differences to exercise the rights on information and consultation are also considered, all in order to determine the most suitable representative organ in each case

Secondly, related to the public framework, the right could be so-called representative and institutional participation, as it is performed in different administrative organs and sectorial foundations, the most representative trade unions as responsible for a proper exercise.

Key words: Labour risks prevention; right to participation; right to information and consultation; general and specific representation.

\section{INTRODUCCIÓN}

El derecho de participación legalmente reconocido a los trabajadores en diferentes aspectos relacionados con la organización general de la empresa, y en particular los relativos a la prevención de riesgos laborales, presenta una estructura compleja al tiempo que alcanza distintos ámbitos de actuación (Cuadro 1).

Así en lo tocante al funcionamiento interno de cada unidad de producción (ámbito participativo privado o interno), en el cual existe una dualidad representativa, habiendo de referirse, por un lado, la participación directa e individualizada de cada trabajador, aplicable a empresas de reducidas dimensiones (de hasta 6 trabajadores) y, junto a aquélla, la participación indirecta colectiva y representativa, idónea para empresas de mayores dimensiones (cuando exceden de 10 trabajadores en plantilla).

Ambas posibilidades están inscritas bajo un modelo participativo que bien podría ser definido o calificado como "procedimental", en tanto son los trabajadores destinatarios y están a la vez legitimados para intervenir en la fase previa a la adopción por el empresario de decisiones relacionadas con todos los aspectos inherentes al adecuado funcionamiento de la empresa ${ }^{1}$, entre ellos, sin duda, la faceta preventiva, 
o cuantas medidas sean precisas en aras de ambientes de trabajo seguros y saludables. El objetivo último no es otro sino lograr un ejercicio trasparente y eficaz del gobierno de la empresa en los aspectos relacionados con la seguridad y salud de los empleados.

A un tiempo, el entorno participativo privado o interno a la entidad incluye a su vez un conjunto de derechos englobados en el más genérico de participación, los cuales vienen a constituirse como elementos de indudable importancia y trascendencia para la efectiva integración de los trabajadores en la dinámica preventiva de las empresas y cuya pretensión es desarrollar y consolidar la tan deseada cultura de la prevención.

Paralelamente existe otro marco de actuación, el público, donde la participación puede calificarse como de representativa o institucional, pues es llevada a cabo en diferentes órganos administrativos y fundaciones sectoriales, siendo los responsables de su ejercicio los sindicatos más representativos.

Cuadro 1: Estructura de la participación de los trabajadores en la empresa en materia de prevención de riesgos laborales

A. Participación en el ámbito privado o interno a la empresa:

A.1. Participación directa e individualizada

(en empresas de seis o menos trabajadores, ejercida directamente por los trabajadores)

A.2. Participación indirecta, colectiva y representativa

(en empresas de más de 10 trabajadores)

- Representación general:

- Representantes unitarios: Delegados de personal y Comité de empresa

- Representación sindical:

Delegados sindicales

- Representación especializada:

- Delegados de prevención

- Comité de seguridad y salud

B. Participación en el ámbito público:

B.1. Participación representativa e institucional

(a través de los sindicatos más representativos)

Fuente: Elaboración propia. 


\section{LA PARTICIPACIÓN EN EL ÁMBITO PRIVADO E INTERNO A LA EMPRESA: LA TITULARIDAD JURÍDICA DEL DERECHO DE PARTICIPACIÓN EN MATERIA PREVENTIVA}

Entre los cauces complementarios para materializar la integración participativa de los operarios en las políticas preventivas de sus respectivas unidades de producción, ha de hacerse necesariamente mención a la "formación" e "información", así como a los de "consulta" y "propuestas".

Por otro lado, la titularidad jurídica del derecho de participación en el ámbito privado e interno a la empresa en materia de prevención de riesgos debe ser considerado bajo una doble dimensión. Así, y por un lado, cabe deducir la existencia de determinados derechos englobados genéricamente en el término "participación", cuya titularidad es claramente subjetiva ${ }^{2}$, concerniente a cada trabajador individualmente considerado, pero cuyo ejercicio va a precisar en ocasiones -y por ello va a adoptar-forma colectiva ${ }^{3}$.

2.1. La titularidad jurídica individual del derecho de participación en materia preventiva

Bajo el punto de vista de la "concepción individual del derecho", las de formación e información son, sin lugar a dudas, algunas de las principales acciones en las cuales mejor queda reflejada la faceta individual de la participación de todos los trabajadores ${ }^{4}$.

Encontrar respaldo a la previa afirmación resulta relativamente fácil. Para ello baste recordar, sin ir más lejos, cómo cada uno de los operarios y a título individual debe ser oportunamente formado e informado en materia preventiva, tal cual especifican los arts. 18 y 19 de la Ley de Prevención de Riesgos Laborales (LPRL) de cara a un adecuado y a la vez seguro desenvolvimiento de sus funciones, y esto sean cuales sean las tareas a realizar y sea cual sea el sector productivo en el que se encuentre encuadrada la empresa.

2 Tal y como indica, entre otros, Valdés Dal-Ré (1997: 73).

3 Por el contrario, Montoya Melgar y Pizá Granados (1996: 246).

4 Como indica Gómez Etxebarría (2007: 87), dentro de esta visión individual debe ser también integrado el trabajador autónomo, en tanto titular de tales derechos (información, consulta y participación). 
Donde realmente mayores dificultades de ejecución plantea la participación individual es en relación al derecho de "consulta" (art. 33 LPRL), puesto que aquí cambia sensiblemente la situación con respecto a la que acaba de ser mencionada. En esta ocasión, el ejercicio del derecho de consulta podría complicarse sobremanera en caso de tratar de proceder a su realización personalizadamente ${ }^{5}$, salvo en el supuesto concreto de aquellas entidades empresariales de pequeñas dimensiones ${ }^{6}$, donde presumiblemente el empresario se entiende -al menos a priorimás accesible para los trabajadores. Bajo un supuesto contrario, esto es, el caso de empresas de mayores dimensiones, el derecho deberá en realidad instrumentalizarse a través de los representantes de los trabajadores (arts. 33.2 y 34.1 LPRL) ${ }^{7}$.

\subsection{La titularidad jurídica representativa del derecho de} participación en materia preventiva

En segundo lugar, pero no por ello menos importante, estaría la dimensión más colectiva del derecho, al ser expresamente otorgada la autonomía colectiva a las instancias representativas de los trabajadores en el seno de la empresa ${ }^{8}$. Dicha autonomía, a su vez, va a estar condicionada por las características estructurales y el tamaño de las empleadoras, lo cual viene a decir que se materializará según corresponda en cada tipo de empresa ${ }^{9}$, a través de sus órganos de representación generales ${ }^{10}$ (ya sean los representantes unitarios del personal -comité de empresa y delegados de personal-), o a través de los representantes sindicales (delegados sindicales $)^{11} \mathrm{y}$, sobre todo, a través de la representación especializada,

5 En este sentido, y entre otros, Fernández Marcos (1997: 196). Obviamente, el empresario no puede consultar con todos y cada uno de los trabajadores las decisiones a adoptar en materia de prevención -y, más en concreto, en cuanto concierne a los aspectos formativos-, decantándose además por la representación específica sobre la materia (los delegados de prevención y el comité de seguridad y salud).

6 Entre otros, Narocki Flaminman (1997: 171), Martínez Girón (1999: 841), Cruz Villalón (2003: 35) y Tascón López (2008: 126 y ss.).

7 A este respecto, véase Cabeza Pereiro (1998: 239-249).

8 Garrido Pérez (1999: 213-224).

9 Agra Viforcos (2005: 23-24). También, Garrido Pérez (1997: 36).

10 Meléndez Morillo-Velarde (2004b: 111). También, Samper Juan (2006: 212-218).

11 Ahora bien, quedando los sindicatos (como tales) expresamente excluidos del colectivo de sujetos con derecho a ser consultados e informados. Así es corroborado en diferentes sentencias, como la del Tribunal Supremo (STS) de 6 de mayo de 1998 (RJ 4099), o la del Tribunal Superior de Justicia de Madrid (STSJ Madrid) de 7 de febrero de 2005 (Rce. 197/2005). 
cuales son los delegados de prevención ${ }^{12}$ (art. 35 LPRL) y el comité de seguridad y salud ${ }^{13}$ (art. 38 LPRL).

$Y$ al igual que los trabajadores individualmente considerados, sus representantes también deben ser formados e informados convenientemente. El derecho de consulta reconocido en el art. 33 LPRL menciona expresamente cómo "en las empresas que cuenten con representantes de los trabajadores las consultas a que se refiere el apartado anterior se llevarán a cabo con dichos representantes". De este modo se está otorgando un mayor peso específico al derecho de consulta con vistas a su ejercicio a través de los representantes, convirtiéndose de alguna manera dicho precepto en el nexo de unión entre las dimensiones individual y colectiva.

2.3. La organización y ejecución de la participación de los representantes de los trabajadores en materia preventiva

Este nivel de participación en la empresa debiera ser calificado como de "privada"14, "indirecta", "colectiva" y "representativa". Tales apelativos resultan justificados en el sentido de no ser una participación ejercida de forma directa y personal por cada trabajador (esto es, individual y directamente) con respecto a su correspondiente patrono, sino conjunta y colectivamente, de forma indirecta, a través de los órganos y estructuras de representación con competencias sobre la materia ${ }^{15}$.

De este modo, y en virtud del tratamiento dado en la LPRL, cabe ver cómo en relación con este modelo participativo en materia preventiva y de protección de la seguridad y salud laboral son planteadas varias alternativas posibles de cara a su ejercicio material. Cada una de ellas, a efectos prácticos, viene apriorísticamente y con carácter mutuo a

12 Teniendo éstos una serie de competencias -por una parte genéricas- en términos de participación, así como de consulta, de vigilancia y control, e informativas. Véase, en este sentido, Purcalla Bonilla (1998: 163-164).

13 Así parece entenderlo, entre otros, Fernández Marcos (1995: 827; 2006: 189). En el mismo sentido, Valdés Dal-Ré (1997: 73). Murcia 2007: 41).

14 "Por realizarse intramuros de la correspondiente relación privada" (García

15 Véase, en este sentido y entre otros, Aguilera Izquierdo (2000: 1240). También, Rodríguez-Sañudo Gutiérrez (1979: 417), De la Villa Gil (1980: 20) y Rodríguez Hidalgo y Álvarez Cuesta (2004: 17). 
constituirse complementaria de las otras, si bien resulta apropiado plantear su análisis bajo dos aspectos bien diferenciados.

Así, en un primer plano está el carácter "orgánico" impregnando la actividad participativa, es decir, el preciso para su ejercicio, debiendo disponerse y contar con determinados órganos de representación para poder llevarla a efecto. Por su parte, y en segundo lugar, el aspecto más "funcional" de la misma, relativo a las facultades y competencias a desempeñar, así como a los requisitos necesarios para su desempeño ${ }^{16}$.

Evidentemente, el análisis propuesto se refiere a las entidades cuyas plantillas exceden en número de los mínimos previstos para la participación directa (unidades de producción con número de trabajadores suficiente para elegir a sus representantes). Sólo partiendo de esta premisa procede el examen de la estructura orgánica y funcional de los diferentes agentes con capacidad para ejercitar la participación colectiva.

2.3.1. Estructura orgánica de la participación representativa de los trabajadores en materia de prevención de riesgos laborales

El reconocimiento del derecho de participación representativa o colectiva no es algo innovador y/o derivado de la aprobación de la Ley de Prevención de Riesgos Laborales (LPRL). Esto es, no supone una inclusión ex novo en la redacción del art. 34 LPRL, pues ya era prevista y regulada en los arts. 4.1 y 6.1 del Estatuto de los Trabajadores (ET), aunque bajo otros parámetros.

Así pues, la regulación contenida en los art. 34 y ss. LPRL no ha supuesto la instauración de nuevos canales de participación, tratándose en realidad de un refuerzo de la representación unitaria preexistente, atribuyéndole competencias participativas más amplias y específicas, acordes con una mejor defensa y protección de la seguridad y salud de los trabajadores.

La estructura participativa preventiva está orgánicamente integrada o puede llevarse a cabo, por un lado, a través de los órganos de

16 Entendido éste en relación al ejercicio, por parte de los diferentes órganos de representación (general o especializada), de determinadas funciones o cometidos de muy diversa naturaleza, entre los cuales pueden contarse los de índole formativa (Valdés Dal-Ré 1997: 85). 
"representación general" (esto es, la representación unitaria -comité de empresa y delegados de personal-), así como a través de los representantes sindicales (pero no los sindicatos); por otro lado, vía "representación especializada" (delegados de prevención y comité de seguridad y salud). Ahora bien, lo anterior suscita una eventual cuestión acerca de cuál o cuáles de entre los posibles órganos de representación referenciados debe desempeñar en cada caso la labor participativa en materia preventiva dentro del tejido empresarial español.

En tal sentido, a priori podría resultar tentador decantarse simple y llanamente por la "especialidad". Ahora bien, analizados diferentes posicionamientos doctrinales y judiciales sobre esta particular cuestión, y al igual que respecto a otros muchos aspectos jurídicos del ordenamiento interno español, se constata la no unanimidad de pareceres.

Así, y en primer lugar, un sector doctrinal es partidario de considerar la complementariedad y la compatibilidad de ambas opciones de manera simultánea, no siendo en ningún momento la una excluyente de la otra ${ }^{17}$. Esto es, en el supuesto de existir representantes de ambas modalidades (representación general y representación especializada), bien sea dentro de una misma empresa o un mismo centro de trabajo -nada infrecuente, por otra parte-, ambas unidades de representación serían igualmente competentes de cara a la participación en materia preventiva, pero con una particular peculiaridad en el caso de cada una de ellas, en el sentido de articular su actuación de acuerdo con las respectivas normas legales de regulación de sus funciones y competencias (ya sea en el ET, la Ley Orgánica de Libertad Sindical -LOLS- o la propia LPRL).

Otra parte de la doctrina iuslaboralista, aun sin negar la doble posibilidad representativa sobre la materia, opta por considerar la representación general como "limitada" y "residual", descartando de este modo la hipótesis de utilización por parte del legislador de las expresiones de ambas modalidades representativas como sinónimas la una de la otra. Tal corriente de opinión prefiere decantarse por la mayor idoneidad de los representantes especializados (los delegados de prevención y el Comité

17 En tal sentido, Sala Franco (2007: 199-200) lo justifica en torno al "y" copulativo utilizado por el legislador en la redacción de la norma, lo cual y según este autor, viene a significar claramente que en el caso de existir representación genérica y especializada de los trabajadores dentro de una determinada empresa o centro de trabajo, ambas representaciones serán competentes en materia de prevención. 
de Seguridad y Salud) ${ }^{18}$, atribuyéndoles la cualidad de representación en relación con la materia concreta de prevención, pues, al menos a primera vista, son los mejores conocedores de las distintas situaciones por las cuales atraviesa cada empresa en cuestiones de seguridad y salud, así como acerca de las posibles carencias existentes ${ }^{19}$.

A la par, y no obstante lo anterior, tampoco carece de sentido y resulta coherente la argumentación de quienes son partidarios de defender la "complicidad" de ambas estructuras participativas ${ }^{20}$, pues en cualquier caso "es difícil de imaginar la instauración" de representaciones especializadas al margen de las representaciones generales, habida cuenta de deber ser elegidos los delegados de prevención por y entre los representantes del personal (esto es, los representantes unitarios y sindicales, según indica el art. 34 LPRL) en el ámbito de los órganos de representación previstos en las normas (art. 35 LPRL). Cabe apreciar cómo, de algún modo, el sistema de representación especializada en realidad está asentado sobre la base de la representación general existente en cada caso ${ }^{21}$.

18 Véase, en este sentido, Peña Obiol (1997: 465-474).

19 Entre otros, Valdés Dal-Ré (1997: 86-87) y Quesada Segura (1997b: 50).

20 Sobre los supuestos de concurrencia y superposición de órganos representativos -soportes estructurales de la autonomía colectiva-, en función de las dimensiones de la empresa o del centro de trabajo, ver Purcalla Bonilla (1997: 369).

21 Permitiendo una pluralidad organizativa (Gete Castrillo 1997: 389). Cabe, no obstante, hacer mención a las conclusiones a este respecto de la $\mathrm{V}$ Encuesta Nacional de Condiciones de Trabajo. Al hacer referencia a la forma de organizar la prevención y, más en concreto, el modo de instrumentalizar la participación con referencia a los delegados de prevención y comités de empresa, los datos obtenidos en 2003 indican que "en un 54’7\% de los centros de trabajo de seis o más trabajadores hay algún delegado de prevención de riesgos laborales. Este dato significa un incremento de doce puntos respecto a 1999 y de veintiséis respecto a 1997. Pese a este crecimiento continuado, se trata de una figura que aún está ausente en el $44 \%$ de los centros. Como ya ocurriera en 1997 y 1999, el incremento actual se ha producido, fundamentalmente, en los centros de trabajo con menor plantilla; por otra parte, sigue siendo más frecuente la presencia del delegado de prevención en los centros de más de 50 trabajadores".

Por sectores de actividad económica, la presencia de delegados de prevención es, de forma similar a como ocurría en ediciones previas de la Encuesta, más frecuente en las ramas de industria $(65 \%)$ que en las de servicios (47'7\%). Destacan por su mayor frecuencia, y entre las primeras, los epígrafes de "otras industrias" (70\%) y "metal" (69’5\%). Respecto a 1999, en todas las ramas de actividad, y aunque en mayor o menor medida, han ido incrementándose los porcentajes de centros con delegado de prevención.

En este mismo orden de cosas, conforme a la escala establecida por la LPRL -la cual fija el número de delegados de prevención en función del número de trabajadores-, y según los responsables de las empresa, el 86 '5\% de los centros de trabajo en los cuales ha sido nombrado delegado de prevención, tienen como mínimo el número de éstos exigido por la Ley. No hay diferencias estadísticamente significativas con respecto a la frecuencia obtenida en 1999.

Respecto a su formación, la mayor parte de los encuestados afirman que los delegados de prevención de su centro han recibido formación sobre seguridad y salud en el trabajo 
Con todo, las concesiones dispositivas para la determinación de la organización representativa más idónea no acaban ahí, facultando además la propia LPRL a la negociación colectiva para acordar, en su caso, la posibilidad de ejercer las competencias conferidas por ella misma a los delegados de prevención "por órganos específicos creados en el propio convenio o en los acuerdos citados" (art. 35.4 LPRL) ${ }^{22}$, es decir, por medio de otros cauces distintos a los delegados de prevención ${ }^{23}$.

\subsubsection{Estructuración funcional de la participación repre-} sentativa de los trabajadores en materia de prevención de riesgos laborales

Procede, pues, analizar las distintas competencias y facultades asignadas a cada una de las organizaciones representativas, tanto la general como la especializada.

En cuanto hace a la representación general, conforme expresa el art. 34.2 LPRL, son las competencias y facultades legalmente asignadas en

desde su designación, bien sea todos o algunos de ellos. En este sentido, los resultados de 2003 muestran un incremento estadísticamente significativo respecto a 1999, pasando de un $88 \%$ a un $94 \%$.

Podría resultar interesante contrastar todas estas conclusiones con las obtenidas a partir de los resultados de la VI y última Encuesta Nacional de Condiciones de Trabajo, según la cual, y en relación con la opinión de los trabajadores acerca de la organización de la prevención (pues, a diferencia de lo ocurrido en la edición previa, en la cual eran entrevistados trabajadores y responsables de las empresas, en ésta última son considerados por separado los puntos de vista de uno y otro colectivos), tan sólo el $55 \%$ de éstos manifiesta haber sido designado delegado de prevención en su centro de trabajo. En las empresas de mayor tamaño ese porcentaje alcanza el $85 \%$, siendo más frecuente la respuesta positiva de los trabajadores del sector industrial y, dentro de éste, de los de las ramas de química y metal. Por su parte, el comité de seguridad y salud estaría constituido en la mayor parte de los centros de trabajo de más de 50 empleados. El 30\% de los entrevistados no sabía de qué recursos preventivos disponía su empresa, siendo el más frecuentemente señalado entre quienes respondieron afirmativamente el servicio de prevención ajeno, seguido por el servicio propio (este último, lógicamente, más frecuente cuanto mayor es el tamaño de la organización).

22 Según Quesada Segura (1997a: 360), serían normas instrumentales, es decir, de un tipo de medidas previstas por la Ley las cuales permiten a la negociación colectiva articular de manera diferente los instrumentos de participación de los trabajadores respecto de las cuestiones relacionadas con la protección de la seguridad y salud de éstos en sus respectivas empresas. De alguna manera, la pretensión no es otra sino la de evitar la destrucción de aquellas experiencias positivas de regulación convencional avanzadas y, sobre todo, de las adaptadas a las necesidades de los sectores productivos a los cuales se dirigen, habiendo demostrado su utilidad, por cuanto pudieran llegar a perderse como consecuencia de ser sustituidas por otras reglas más rígidas planteadas en la norma legal, hecho éste el cual queda salvaguardado por la Disposición Transitoria de la propia LPRL.

23 En este sentido, véase ampliamente Valdés Dal-Ré (1997: 93-94), argumentando cómo, a través de la redacción hecha del art. 35 LPRL no sólo está autorizándose a la negociación colectiva a crear y/o cambiar la forma de designar a los representantes especialistas, o incluso a proceder a la elección de otros órganos distintos de representación, sino además otorgándosele la facultad de cambiar tanto el cuerpo de electores (art. 35.4) como el colectivo de elegibles (art. 35.2), sin incorporar en tal caso la norma legal (la LPRL) en su articulado restricciones de ningún tipo. De este modo, delegado de prevención podrá ser un trabajador no representante, o un representante no unitario, o cualquier otro posible tipo de combinación pactada en un convenio colectivo. 
sus respectivas normas de procedencia, el ET y la LOLS, correspondiendo en cualquier caso a los representantes unitarios y representantes sindicales "la defensa de los intereses de los trabajadores en materia de prevención de riesgos en el trabajo" (art. 34.2 LPRL), y "ejercer una labor de vigilancia y control sobre el cumplimiento de la normativa de prevención de riesgos laborales" [art. 36.1.d)] durante el desarrollo del trabajo en la empresa, con las particularidades previstas en este orden por los arts.18 y 19.4 ET, entre las cuales han de destacarse significativamente los aspectos relacionados con la obligación de formación teórica y práctica y la información, a las cuales está sometido el empresario en el ámbito de la seguridad e higiene en el entorno laboral.

No obstante, tal y como cabe apreciar con toda claridad, el contenido de la LPRL únicamente se limita a recordar las competencias asignadas por el legislador a los órganos integrantes de esta modalidad representativa genérica en lo referente a la materia de seguridad y salud laboral, apareciendo éstas, a su vez, recogidas en sus respectivas normas de referencia; más concretamente a través de los arts. 64, 65 y $87 \mathrm{ET}^{24}$, recientemente modificados (a lo cual se hará referencia con posterioridad), y de los arts. 8.2 y 10.3.1 LOLS $^{25}$.

También es imprescindible hacer alusión en este apartado a la participación a través de la representación especializada, cuyos mecanismos de acción pueden concretarse en dos, cuales son los delegados de prevención, figura nuclear sobre la cual se asienta el sistema de representación especializada en materia preventiva ${ }^{26}$ (arts. 35 a 37 LPRL), y el comité de seguridad y salud (arts. 38 y 39 LPRL).

Respecto de los delegados de prevención, y al margen de las múltiples peculiaridades propias de su elección (materia ésta cuyo detalle no es objeto de las presentes páginas), debe recordarse la posibilidad de ser elegidos, conforme establece el art. 35.1 LPRL, por y de entre los

24 Art. 64.4.d) ET, en materia de consulta (incluida la emisión de informes); art. 64.8 ET, en materia de información; arts.64.9 y $65.1 \mathrm{ET}$, en materia de vigilancia y control, así como para el ejercicio de acciones ante la empresa y los tribunales competentes; y art. 87.1 ET, en materia de negociación colectiva, es decir, sobre representación y participación institucional y a la vez pública.

25 Art. 8.2 LOLS, en cuanto a la participación institucional a través de la negociación colectiva; art. 10.3.1 LOLS, en cuanto a la información a la cual tienen derecho sobre aspectos concretos del funcionamiento de cada entidad productiva, es decir, en cuanto a conocer con carácter trimestral datos estadísticos sobre absentismo, accidentes, etc.

26 Durán López, Tudela Cambronero y Valdeolivas García (2008: 263). 
representantes del personal, a la vez que estando, por otro lado, también reconocida la capacidad de la negociación colectiva para establecer otro sistema de designación (art. 35.5 LPRL), si bien y a la postre todo queda interrelacionado, pues con harta frecuencia los encargados de la negociación son también los mismos representantes unitarios y sindicales antes aludidos. Todo ello amén de la ya avanzada y adicional posibilidad adicional consistente en el establecimiento de otro tipo de órganos específicos por acuerdo en el propio convenio colectivo (párrafo segundo art. 35.5 LPRL) ${ }^{27}$.

Pero lo realmente relevante y significativo en cuanto aquí atañe, son las competencias y facultades a estos representantes asignadas, las cuales aparecen relacionadas en el art. 36 LPRL, incluyendo la adopción de las medidas necesarias y más idóneas para garantizar adecuadamente la seguridad y salud de los trabajadores. Entre ellas desempeña evidentemente un papel destacado el apartado dedicado a la formación, en relación con la cual es preciso diseñar un programa eficaz, debiendo participar activamente los delegados de prevención en tanto parte directamente implicada, al disponer de información de primera mano acerca de las posibles carencias o deficiencias detectadas en los propios escenarios en los cuales son generadas.

En este sentido, el legislador ha venido realizando un importante esfuerzo por introducir la especialización de un segmento de la representación de los trabajadores a cuyos integrantes el empresario ha de formar convenientemente ${ }^{28}$. Tal grado de instrucción es el requerido para poder atribuirles una serie de funciones, entre las cuales brilla con luz propia la faceta formativa de los operarios de cada empresa en particular, y ello entendido tanto en términos de impartición directa como de diseño de la misma.

Una vez establecidas las estrechas interrelaciones entre una y otra forma de representación, surge un nuevo interrogante, concerniente a la determinación en cuanto a la coincidencia o no de las competencias y facultades asignadas a los posibles órganos de representación (general y especializada). Sobre este particular, y si bien en un primer momento quizá todo apuntara hacia una coincidencia de funciones, al profundizar sobre tal extremo bien podría llegarse a otra posible apreciación.

Franco (2007: 201-206)

27 Considerada, entre otros, por Valdés Dal-Ré (1997: 93). También, por Sala

28 Sobre los medios y cualificación adecuados, véase Moreno Cáliz (2005: 405). 
Así pues y aun cuando el principio de especialización funcional es el que actúa como eje principal de la política legislativa, informando el derecho de participación de los trabajadores en materia de seguridad y salud laboral, bien puede afirmarse la circunstancia de de no haber sido llevado por la LPRL hasta sus últimas consecuencias.

Por tal motivo, aunque ambos grupos representativos (generales y especializados) ostentan competencias sobre la materia de protección de la seguridad y salud laboral), las actuaciones de unos y otros atienden más a un principio de "división", y no tanto a uno de "concurrencia" funcional ${ }^{29}$.

Tal es así, que en el transcurrir cotidiano de las empresas viene siendo habitual la reserva a través de la negociación colectiva de las funciones más típicamente "reivindicativas" en lo concerniente a las acciones preventivas a la representación general en su faceta de actuación pública ${ }^{30}$, habida cuenta de ser los encargados de reivindicar y establecer las pautas de actuación, concretando, entre otros extremos, el contenido, la duración, el momento y el lugar para la impartición de formación, junto con otros muchos detalles. No obstante, esto no siempre se hace, siendo aún abultado el número de convenios que se limitan únicamente a reproducir las exigencias establecidas en la norma ${ }^{31}$.

De este modo, bien pudiera resultar factible la concurrencia funcional en algunos aspectos relacionados con la prevención, tales como, por ejemplo, las funciones de vigilancia del cumplimiento de las obligaciones preventivas de formación para un adecuado manejo de los equipos de trabajo y de los equipos de protección -tanto los individuales como los colectivos-, así como con vistas al adecuado manejo y manipulación de las materias primas, entre otras muchas.

Por otro lado, estaría la asignación de aquellas otras funciones cuya naturaleza es esencialmente "cooperativa" a los representantes especializados (delegados de prevención y comité de seguridad y salud). En este sentido, y salvo la coincidencia material sobre una misma persona de ser delegado de personal con capacidad para negociar un convenio

29 Valdés Dal-Ré (1997: 96).

30 Pero no siendo, en todo caso, algo ni vedado ni incompatible para la representación especializada (Quesada Segura 1997b: 50).

31 Debiera, no obstante, tomarse en consideración el reparto de competencias entre convenios de distintos ámbitos, en relación a las posibles materias negociables. Véase, en este sentido, Meléndez Morillo-Velarde (2004a: 128-129). 
colectivo y ser a su vez delegado de prevención ${ }^{32}$, estos últimos no tienen en sí mismos capacidad negocial a nivel convencional, a través de la cual en todo caso podría mejorarse el cuadro legal de competencias y facultades, que tienen el carácter de mínimos.

Dada la situación, cabe lógico pensar como parte de las funciones y competencias "especializadas" la impartición, incluso material, de la formación y, por supuesto, de la información sobre la materia de seguridad y salud ${ }^{33}$, reservando la capacidad negociadora para la representación general, la unitaria y también la sindical, principalmente para establecer pautas relativas al contenido de la misma.

Donde realmente es detectada una significativa diferencia funcional entre ambas formas de representación (general y especializada), ésa está estrechamente vinculada con el derecho y la correlativa obligación de "consulta". Si bien es verdad el carácter no vinculante de las propuestas de unos u otros para la toma de decisiones del empresario en relación con esta materia, y aunque es cierto que la modificación producida a raíz de la nueva reorganización establecida a través del punto 5 art. único Ley $38 / 2007$, de 16 de noviembre (el cual modifica el art. 64 ET como consecuencia de la transposición al derecho interno español de la Directiva 2002/2004/CEE, de 11 de marzo de 2002, del Parlamento Europeo) contribuye sin duda a acortar distancias entre ambos tipos de representación al haber variado el tratamiento dado al derecho de consulta con respecto a la representación general, aún siguen existiendo diferencias apreciables.

Así, y cuando la representación general (representación unitaria y sindical) elabora informes y propuestas de índole preventivo en base a las informaciones recibidas del empresario, y los presenta ante el mismo, tras la nueva redacción del art. $64 \mathrm{ET}$, le es reconocido el derecho a reunirse con éste (para quien resulta obligatorio) y a obtener del mismo "una respuesta justificada", así como a contrastar los puntos de vista u opiniones de ambas partes con objeto, en su caso, de poder llegar a acuerdos (art. 64.6 ET). Esto supone un cambio respecto a la situación

32 No siendo, por tanto, incompatible realizar la función cooperativa con otra más reivindicativa de los delegados de prevención, "en cuanto que, en definitiva, son a la par representación especializada y general" (Quesada Segura 1997b: 50).

33 Respecto a las facultades informativas de los delegados de prevención, véase Purcalla Bonilla (1998: 165). 
anterior, pues hasta ese momento las propuestas planteadas podían ser desechadas sin más.

Por su parte, y con respecto de la representación especializada, "la decisión negativa del empresario a la adopción de las medidas propuestas por los delegados de prevención", a tenor del contenido establecido en el art. 36.2.f) LPRL, habrá de ser motivada.

Tal exigencia obliga al empresario, por un lado, a prestar atención a las propuestas, y a no poder limitarse a desecharlas sin más. Por otra parte, existe la posibilidad de una negativa a tenerlas en cuenta, la cual tan solo podrá estar fundamentada en motivos de índole exclusivamente técnica, bien sea demostrando la inexistencia de una real o previsible situación de riesgo, o bien la inadecuación de la medida propuesta a la situación planteada o susceptible de plantearse $y$, en todo caso, dando lugar asimismo a interpretar como no válidas a estos efectos cualesquiera motivaciones de carácter únicamente económico, productivo o de interés organizativo ${ }^{34}$.

Otro de los aspectos funcionales que presenta alguna que otra diferencia en cuanto al tratamiento de unos $u$ otros grupos representativos es el relativo al derecho de "información", por cuanto, y si bien todos lo tienen reconocido, el alcance y repercusión para unos y otros en materia preventiva difiere sensiblemente.

\section{a) Respecto de la representación general}

El derecho de información reconocido a la representación general presenta una doble perspectiva. Por un lado, está el derecho de "información activa", reconocido a los representantes de los trabajadores al objeto de poder informar tanto al resto de los trabajadores -sus representados- como a la propia empresa, y a cuyos efectos deberán disponer de los instrumentos materiales necesarios para un correcto

34 Véase, en este sentido, Quesada Segura (1997b: 59). La autora va más allá en su análisis, tratando de establecer las posibles soluciones ante negativas del empresario injustificadas o no suficientemente motivadas, llegando a la conclusión de, además de las correspondientes acciones administrativas o de otro tipo susceptibles de ser ejercidas por parte los representantes legales de los trabajadores, en caso de dar lugar a efectos adversos para la salud de los operarios implicados, éstos podrían incluso recurrir a la vía de la Inspección de Trabajo, y ello en virtud de la previsión contenida en el art. 40.1 LPRL, cuyo tenor determina la posibilidad de exigir responsabilidades "si consideran que las medidas adoptadas y los medios utilizados por el empresario no son suficientes para garantizar la seguridad y salud en el trabajo". 
ejercicio del mismo, tablones de anuncios y medios informáticos adecuados para facilitar la labor.

En segundo lugar queda situada la "información pasiva", identificada con el contenido informativo facilitado por la empresa, y donde "los representantes de los trabajadores son meros receptores de los datos suministrados por la empresa"35, y ello aun cuando pueda tratarse de datos concretos legalmente reconocidos, los cuales "no satisfacen íntegramente las necesidades de información que exige el pleno ejercicio de sus facultades de vigilancia y control".

Esta situación ha sido objeto de modificación en términos de efectividad o nivel de exigencia, pues hasta la entrada en vigor de la Ley 38/2007 existía una escasa cobertura coercitiva para los supuestos de incumplimiento por parte del empresario de la obligación de información, dando lugar hasta entonces únicamente a una infracción administrativa, contemplada en el art. 7 del texto refundido de la Ley sobre Infracciones y Sanciones en el Orden Social, LISOS (considerando tal incumplimiento como falta grave sancionable con una multa de cuantía entre 300 y 3.000 euros).

Como ocurriera con el derecho de consulta, las facetas informativa y consultiva reconocidas a los representantes de los trabajadores a través del comité de empresa han sido objeto de reciente y nueva reorganización, mucho más completa y esclarecedora, a través del punto 5 art. único de la mentada Ley 38/2007, de 16 de noviembre.

Lo más destacable de la nueva redacción del art. 64 ET es el expreso reconocimiento de la información como derecho básico de los trabajadores ${ }^{36}$, en los siguientes términos: "el comité de empresa tendrá derecho a ser informado y consultado por el empresario sobre aquellas cuestiones que puedan afectar a los trabajadores, así como sobre la situación de la empresa y la evolución del empleo en la misma...". Queda así automáticamente incluida la información sobre cuantos aspectos puedan afectar a la seguridad y a la salud de los trabajadores, así como al respecto de las medidas preventivas y protectoras a adoptar.

También es proporcionada una definición en relación al alcance y significado del término "información", en este concreto contexto

\footnotetext{
35 Samper Juan (2006: 257).
}

36 Sempere Navarro (2008: 12). 
entendida como "la trasmisión de datos por el empresario al comité de empresa, a fin de que éste tenga conocimiento de una cuestión determinada y pueda proceder a su examen" (punto 5.1. art. único Ley 38/2007 y art. 64.1 ET en su nueva redacción) ${ }^{37}$.

En el proceso informativo deben primar tanto por una como por otra de las partes implicadas -esto es, el empresario y el comité de empresa- el compromiso y la obligación de regirse por un espíritu de cooperación, dando de este modo adecuado cumplimiento a los recíprocos derechos y obligaciones sobre la materia, y a la vez debiendo respetar en todo momento tanto los intereses de la empresa como los de los trabajadores. Todo ello, no obstante, sin perjuicio de la posibilidad de remitir a la negociación colectiva la definición de las diferentes modalidades prácticas de ejecución de la acción de información (novena competencia del art. 64.7 ET).

El derecho de información queda estructurado conforme al contenido con diferentes frecuencias. En primer lugar, y con carácter trimestral, el empresario debe informar sobre diferentes aspectos, entre los cuales merece ser destacado el tocante a "los accidentes de trabajo y enfermedades profesionales y sus consecuencias, los índices de siniestralidad, los estudios periódicos o especiales del ámbito laboral y los mecanismos de prevención que se utilicen" (punto 5.2. art. único Ley $38 / 2007$ y art. 64.2 ET). Esto amén de otros aspectos de igual importancia, pero no tan íntimamente relacionados con la prevención de riesgos.

Del mismo modo y carácter, el comité de empresa tiene derecho a ser informado trimestralmente sobre la situación y estructura del empleo en la entidad, pero sobre todo acerca de "las decisiones de la empresa que pudieran provocar cambios relevantes en cuanto a la organización del trabajo y a los contratos de trabajo en la empresa", así como sobre "las eventuales medidas preventivas, especialmente en caso de riesgo para el empleo" (punto 2.3. art. único Ley 38/2007 y art. 64.5 $\mathrm{ET})$.

También, y con carácter al menos anual, el empresario ha de proceder a informar acerca de las medidas adoptadas a fin de lograr una efectiva igualdad entre hombres y mujeres, incluyendo sobre este particular datos concernientes a la proporción de mujeres y hombres en

37 Anticipándose a la Ley y analizando las implicaciones de la norma europea sobre la materia (Palomeque López 2006: 59 y ss.). 
los diferentes niveles profesionales de la organización, debiendo ser incluidas entre dichas medidas las relativas a aspectos relacionados con la seguridad y la salud (punto 5.3. art. único Ley 38/2007 y art. 64.3 ET), pues no en vano en determinadas situaciones han de adoptarse medidas idóneas para salvaguardar la salud de la madre y su hijo durante los períodos de gestación y lactancia.

Además son reconocidas al comité de empresa a través del punto 7 art. único Ley 38/2007 y el art. 64.7.a) $2^{\circ}$ ET determinadas competencias, entre otras las de "vigilancia y control de las condiciones de seguridad y salud en el desarrollo del trabajo en la empresa, con las particularidades previstas" en este orden de cosas en el art. 19 del propio ET.

Al margen de todo lo anterior, es especificado expresamente con respecto a la acción informativa debida por el empresario al comité de empresa, el deber de ser ésta facilitada "sin perjuicio de lo establecido específicamente en cada caso, en un momento, de una manera y con un contenido apropiados", permitiendo así a los correspondientes representantes de los trabajadores poder proceder a su examen de forma adecuada y comprensible y, en su caso, elaborar la oportuna consulta y el correspondiente informe (punto 5.6. art. único Ley 38/2007 y art. 64.6 $\mathrm{ET})$.

\section{b) Respecto de la representación especializada}

Entre las competencias y facultades asignadas a los delegados de prevención se encuentra la de recibir información, apareciendo recogida en dos puntos del articulado de la LPRL, art. 36.2.c) d), poniendo de manifiesto:

[por un lado, su derecho a] ser informados por el empresario sobre los daños producidos en la salud de los trabajadores una vez que aquél hubiese tenido conocimiento de ellos, pudiendo presentarse, aun fuera de su jornada laboral, en el lugar de los hechos para conocer las circunstancias de los mismos; [por otro, a] recibir del empresario las informaciones obtenidas por éste procedentes de las personas u órganos encargados de las actividades de protección y prevención en la empresa, así como de los organismos competentes para la seguridad y la salud de los trabajadores, sin perjuicio de lo dispuesto en el art. 40 de esta Ley en materia de colaboración con la Inspección de Trabajo y Seguridad Social. 
Como puede apreciarse, el contenido de la información a que tienen derecho una y otra clase de representación (general y específica) no debería en esencia diferir sustancialmente, más aún cuando el objetivo último a lograr es alcanzar lugares de trabajo más seguros y saludables.

Analizando, no obstante, jurisprudencia sobre el particular, cabe constatar cómo en la práctica sí existe una diferencia en el tratamiento de unos y otros representantes, tal y como queda patente en STSJ Madrid (Rec. 3867/2006), según la cual los delegados sindicales de una empresa piden el acceso a la misma información y documentación en materia preventiva que el comité de seguridad y salud, siendo desestimada su petición.

motivo:

El argumento justificativo de tal decisión, infiere como

que en ninguno de los apartados del extenso art. 64 del ET se recoge de modo concreto o al menos de manera genérica el derecho postulado por los delegados sindicales actuantes, por lo que su pretensión no aparece legalmente fundada. Para que lo estuviera, hubiera sido necesario haber alegado y probado que la empresa hubiera negado a los delegados sindicales actuantes la misma información y documentación que facilita al comité de empresa, cuestión que ni siquiera ha sido suscitada en la instancia.

En otras palabras, de alguna manera sí se están equiparando los derechos de información de los representantes unitarios entre sí, pero no los considera comparables con el del comité de seguridad y salud, cosa que sí se hizo en primera instancia.

3. LA PARTICIPACIÓN REPRESENTATIVA EN EL ÁMBITO PÚBLICO O INSTITUCIONAL

La participación representativa institucional se encuadra en un ámbito público de actuación, pues en ella quedan implicados como sujetos afectos tanto las organizaciones empresariales y sindicales más representativas como la propia Administración ${ }^{38}$, pudiendo ser identificada en diferentes escenarios.

38 Quesada Segura (1997a: 365-366). 
Esta modalidad representativa viene de algún modo a reforzar el papel de la autonomía colectiva en general, y en materia de prevención de riesgos laborales en particular ${ }^{39}$. Ello se constata cuando en la propia exposición de motivos de la LPRL le es atribuida a la negociación colectiva, al margen de su actuación en los correspondientes entornos profesionales, la facultad de actuar como instrumento de participación de los trabajadores "en ámbitos de actuación distintos a los propios centros de trabajo" ${ }^{40}$. En concreto, a dicha capacidad representativa es reconocida "la necesaria participación en dicha actuación (política preventiva) de empresarios y trabajadores, a través de sus organizaciones representativas". En tal sentido, llegando a lograr una autentica cultura de la prevención.

Así pues, y en primer lugar, es otorgada a la función institucional ${ }^{41}$ de los agentes sociales la consideración de "necesaria" para el adecuado desarrollo de la política en materia de prevención de riesgos laborales llevada a cabo por parte de las Administraciones Públicas en los diferentes niveles territoriales a considerar, ya sea en el ámbito nacional o autonómico, así como en otros supuestos de ámbito inferior ${ }^{42}$.

Dicho reconocimiento queda reflejado en la participación institucional, en los diferentes organismos administrativos creados al efecto por la propia LPRL para un adecuado desarrollo de las políticas de prevención de riesgos y de promoción de la seguridad y salud en los entornos profesionales. Es decir, la faceta pública de la participación comporta la colaboración para la elaboración de las oportunas políticas preventivas, las cuales van a ser llevadas a cabo a través de las organizaciones empresariales y sindicales más representativas (art. 5.1.b LPRL).

De este modo, por ejemplo, los agentes sociales $\mathrm{y}$, por tanto, los representantes de los trabajadores, deben formar parte de la Comisión Nacional de Seguridad y Salud en el Trabajo, órgano colegiado regulado en el art. 13.1 LPRL, cuya finalidad no es otra sino la de realizar una labor de asesoramiento a las Administraciones Públicas en las cuestiones

39 Gete Castrillo (1997: 376 y ss.).

40 En cuanto al establecimiento de la diferencia entre las funciones de la negociación colectiva en los ámbitos privados y públicos, ver Casas Baamonde (1997: 165).

41 "Representación ex lege que normalmente adquiere una dimensión institucional o cuasi pública" (García Murcia 2007: 40-41).

42 González-Posada Martínez (1997: 33-34). 
relativas a la formulación de las políticas de prevención ${ }^{43}$. Según indica el art. 13.2 LPRL, en su composición está integrada por un representante de cada comunidad autónoma, así como un número igual de representantes de la Administración General del Estado, y paritariamente respecto a los citados, representantes en número correspondiente de las organizaciones empresariales y sindicales más representativas.

De entre las muchas atribuciones conferidas por la Ley a citada Comisión cabe señalar, entre otras, conocer "las actuaciones que desarrollen las Administraciones Públicas competentes en materia de promoción de la prevención de riesgos laborales, de asesoramiento técnico y de vigilancia y control" conforme a las previsiones establecidas en los arts. 7 a 11 LPRL. Asimismo "podrá informar y formular propuestas en relación con dichas actuaciones, específicamente sobre otras cuestiones" ${ }^{14}$.

En lo tocante a la toma de decisiones es de destacar que los correspondientes acuerdos habrán de adoptarse por mayoría (art.13.4 LPRL), con la particularidad de otorgar un trato preferente a los agentes sociales, al reconocer a sus representantes dos votos por la parte patronal y dos por parte de los trabajadores, frente a un único voto por parte de cada una de las Administraciones Públicas presentes.

También está prevista la presencia de los representantes de los trabajadores como parte integrante de la Fundación adscrita a la Comisión Nacional de Seguridad y Salud en el Trabajo, siendo sus Estatutos aprobados por ésta (disposición adicional quinta LPRL), cuya finalidad "será promover la mejora de las condiciones de seguridad y salud en el trabajo, especialmente en las pequeñas empresas, a través de acciones de información, asistencia técnica, formación y promoción del cumplimiento de la normativa de prevención de riesgos".

En este mismo orden de cosas, también habrá una participación institucional en las fundaciones de ámbito sectorial, constituidas por empresarios y trabajadores, contándose entre sus fines la promoción de actividades destinadas a la mejora de las condiciones de seguridad y salud en el trabajo, entre las cuales estará la promoción y

43 Rodríguez-Sañudo Gutiérrez (1996: 256).

44 Tales como criterios y programas generales de actuación, proyectos de disposiciones de carácter general, coordinación de las actuaciones desarrolladas por las Administraciones Públicas competentes en materia laboral, y coordinación entre las Administraciones Públicas competentes en materia laboral, sanitaria y de industria (art. 13.3 LPRL). 
mejora de la formación e información sobre la materia, dada su gran repercusión en la reducción de los índices de siniestralidad, de algunos sectores de actividad en particular ${ }^{45}$.

En cuanto hace al ámbito autonómico de actuación, la participación es contemplada en la disposición adicional duodécima LPRL, con el siguiente tenor: "en las comunidades autónomas, la participación institucional, en cuanto a su estructura y organización, se llevará a cabo de acuerdo con las competencias que las mismas tengan en materia de seguridad y salud laboral".

Junto a todo lo anterior, y en segundo lugar, cabe hacer mención a la preceptiva pero no vinculante consulta previa a la elaboración por parte del Gobierno de los reglamentos de desarrollo de la Ley. De acuerdo con el art. 6 LPRL, "el Gobierno, a través de las correspondientes normas reglamentarias y previa consulta a las organizaciones sindicales y empresariales más representativas, regulará las materias...", debiendo éstas además ser revisadas periódicamente, así como habiendo de incluirse necesariamente entre ellas, y entre otras muchas, la consulta acerca de "la exigencia de un adiestramiento o formación previa o la elaboración de un plan en el que se contengan las medidas preventivas a adoptar" [art. 6.1.c) $[\mathrm{LPRL}]^{46}$.

Un tercer escenario de actuación es el relacionado con la faceta de control y el correspondiente seguimiento de la gestión y el funcionamiento de las mutuas de accidentes de trabajo y enfermedades profesionales, como garantía del buen hacer de éstas (art. 32.1 LPRL).

Finalmente, y aun cuando con un carácter más restringido, al afectar únicamente a las organizaciones sindicales más representativas, quedan situadas aquellas otras competencias relativas a las oportunas consultas para la presentación de determinadas propuestas concernientes a la elaboración y organización de las actividades preventivas, formativas e informativas en determinados ámbitos profesionales ${ }^{47}$.

45 Así pues (Álvarez Montero 2001: 246 y ss.), la participación a través de los comités de seguridad y salud (arts. 38 y 39 LPRL) constituye una clara manifestación de la participación equilibrada reclamada por la norma europea (art. 12 Directiva Marco).

46 De acuerdo con Rodríguez-Sañudo Gutiérrez (1996: 256), cabe deducir para la participación, cuando ésta es ejercida en ámbitos o niveles superiores al de la empresa y del centro de trabajo, la no utilización de un mecanismo representativo ad hoc o específico, sino el recurso a otros más generales, cuales son los sindicatos.

47 Quesada Segura (1997a: 367). 
Tales competencias encuentran amparo en la disposición adicional octava LPRL, la cual atribuye la obligación a cada Departamento Ministerial de consulta previa con las organizaciones sindicales más representativas antes de elevar al Consejo de Ministros una propuesta de acuerdo, en la cual sea establecido un plan de trabajo adecuado para la organización de la actividad preventiva en el correspondiente departamento, así como en los centros, organismos y establecimientos de todo tipo dependientes del mismo.

\section{A MODO DE RESUMEN Y CONCLUSIÓN}

Como conclusión de lo expuesto, dos son las posibles formas de ejercitar la participación en materia preventiva en las empresas 0 , dicho de otro modo, existe una doble vía para canalizar el ejercicio del derecho de participación en materia de seguridad y salud laboral en las entidades empresariales (art. 33 LPRL): la individual y la colectiva.

Evidentemente, y a efectos prácticos, cabe deducir la íntima unión entre ambas formas de representación, "general" y "especializada", con difíciles visos de separación desde un punto de vista orgánico. Su nexo de unión radica en la autonomía colectiva, responsable última de la elección de la representación especializada, pues aun cuando no se opte por la designación de los delegados de prevención de entre los representantes unitarios o sindicales, a la postre estos sí son quienes van tener la última palabra al respecto por la facultad a ellos conferida a través de la negociación colectiva, artífice último en la realidad para su designación, al ser parte en la negociación.

La estructura funcional de la participación presenta un reparto tácito de funciones, asignándose en términos generales la faceta más reivindicativa a la representación general y la más cooperativa para la representación especializada.

Además, y como consecuencia de la transposición de la norma europea al ordenamiento interno español por medio de la Ley $38 / 2007$ de 16 de noviembre, los derechos de información y consulta de la representación general, integrados en otro más amplio cual es el de participación, se han visto reforzados. 
BIBLIOGRAFÍA

AGRA VIFORCOS, B. (2005) "Participación y representación de los trabajadores en materia de prevención de riesgos laborales. Especial referencia a las previsiones al respecto del contenido en los convenios colectivos de Castilla y León", Revista de Investigación Económica y Social de Castilla y León, 8, pp. 15-109.

AgUILERA IzQUIERDO, R. (2000) "Instituciones de participación. En especial, delegados de personal (arts. 61 y 62)", Revista Española de Derecho del Trabajo, 100-2, pp. 1239-1254.

Álvarez MONTERo, A. (2001) El delegado de prevención. Un estudio crítico de su régimen jurídico. Granada: Comares.

Cabeza Pereiro, J. (1998) "Art. 33. Consulta de los trabajadores". J. CABezA Pereiro y J.F. Lousada AROCHetena (coords.) Comentarios a la Ley de Prevención de Riesgos. Granada: Comares, pp. 239-250.

CASAS BAAMONDE, M.E. (1997) "Derecho público y salud laboral: el régimen jurídico sancionador". M.E. CASAS BAAMONDE, M.C. PALOMEQUE LóPEZ y F. VALDÉS DAL-RÉ (coords.) Seguridad y salud en el trabajo. El nuevo derecho de prevención de riesgos profesionales. Madrid: La LeyActualidad, pp. 127-181.

CRUZ VILLALÓN, J. (2004) "Las relaciones laborales en la pequeña empresa: una aproximación a sus especialidades normativas". J. CRUZ VILALÓN y F. FUENTES RODRÍGUEZ (coords.) Las relaciones laborales en la pequeña empresa. Cádiz: Universidad de Cádiz, pp. 9-46.

DuRÁN LÓPEZ, F.; G. TUdela CAMBRONERO y Y. VALDEOLIVAS GARCíA (2008) Informe sobre la situación de la prevención de riesgos laborales en el sector de la construcción en España. Madrid: Edisofer.

FERNÁNDEZ MARCOS, L. (1995) "El contenido de la Ley de Prevención de Riesgos Laborales: puntos críticos", Actualidad Laboral, 3, pp. 817-841.

- (1997) "Artículo 33. Consulta de los trabajadores". M. IgLESIAS CABERO (coord.) Comentarios a la Ley de Prevención de Riesgos Laborales, Madrid: Civitas, pp. 195-197.

- (2006) Comentarios a la Ley de Prevención de Riesgos Laborales y su régimen jurídico sancionador, $4^{\text {a }}$ ed. Madrid: Dykinson.

García MuRCIA, J. (2007) "Los convenios colectivos como fuente de la relación laboral: más apuntes para un debate recurrente", Revista del Ministerio de Trabajo y Asuntos Sociales, 68, pp. 25-49. 
GARRIDO PÉREZ, E. (1997) "La participación de los trabajadores en materia de prevención de riesgos laborales en la Ley 31/1995", Relaciones Laborales, 2, pp. 397-427.

- (1999) "Consulta y participación de los trabajadores en materia de salud laboral", Cuadernos de Derecho Judicial, 1, pp. 211-262.

Gete CASTRILlo, P. (1997) "El papel de la negociación colectiva en la prevención de riesgos laborales", La Ley-Actualidad, 1, pp. 371-393.

Gómez EtXeBARRíA, G. (2007) Prevención de riesgos laborales del trabajador autónomo. Bilbao: CISS.

GONZÁLEZ-POSADA MARTÍNEZ, E. (1997) "La política de prevención de riesgos laborales. Objetivos y agentes. El papel de las Administraciones Públicas". M.E. CASAS BAamonde, M.C. PALOMEQUe LóPeZ y F. VAldés Dal-RÉ (coords.) Seguridad y salud en el trabajo. El nuevo derecho de prevención de riesgos profesionales. Madrid: La Ley-Actualidad, pp. 17-34.

LÓPEZ GANDÍA, J. y J.F. BLASCO LAHOZ (2002) Curso de prevención de riesgos laborales: nivel básico, $4^{\mathrm{a}}$ ed. Valencia: Tirant lo Blanch.

MARTÍNEZ GIRóN, J. (1999) "La seguridad y la salud laboral en la pequeña empresa", Actualidad Laboral, 3, pp. 839-845.

MELÉNDEZ MORILLO-VeLARDE, L. (2004a) "Obligaciones de seguridad y salud y negociación colectiva", Revista del Ministerio de Trabajo y Asuntos Sociales, 53, pp. 121-148.

- (2004b) La prevención de riesgos laborales en la negociación colectiva. Pamplona: Aranzadi.

Montoya Melgar, A. y J. PizÁ Granados (1996) Curso de seguridad y salud en el trabajo. Madrid: McGraw-Hill.

MORENO CÁLIZ, S. (2005) "La participación de los trabajadores: los derechos de información y consulta. La representación de los trabajadores para la prevención de riesgos". J.I. GARCíA NINET (dir.) A. GARRIGUES GIMÉNEZ (coord.) Manual de prevención de riesgos laborales (seguridad, higiene y salud en el trabajo), $2^{\mathrm{a}}$ ed. Barcelona: Atelier, pp. 351-386.

NAROCKI Flaminman, C. (1997) "La prevención de riesgos laborales en las pequeñas y medianas empresas españolas", Cuadernos de Relaciones Laborales, 10, pp. 157-181.

PALOMEQUe LóPEZ, M.C. (2006) "La participación de los trabajadores en la empresa (una revisión institucional)", XVII Congreso Nacional de Derecho del Trabajo y de la Seguridad Social. Gobierno de la empresa y participación de los trabajadores: viejas y nuevas formas institucionales, Salamanca: AEDTSS / Universidad de Salamanca), pp. 13-65. 
PEÑA OBIOL, S. (1997) "Algunos aspectos más significativos de la reciente Ley de Prevención de Riesgos Laborales. Una especial referencia a la nueva regulación del Comité de Seguridad y Salud Laboral". R. ESCUDERO RODRÍGUEZ (coord.) XIV Jornadas Universitarias Andaluzas de Derecho del Trabajo y Relaciones Laborales. La Ley de Prevención de Riesgos Laborales. Sevilla: Consejo Andaluz de Relaciones Laborales, pp. 465-474.

PuRCALLA BONILLA, M.A. (1997) "Análisis del papel de la autonomía colectiva en materia de prevención de riesgos profesionales". R. ESCUDERO RODRíGUEZ (coord.) XIV Jornadas Universitarias Andaluzas de Derecho del Trabajo y Relaciones Laborales. La Ley de Prevención de Riesgos Laborales. Sevilla: Consejo Andaluz de Relaciones Laborales, pp. 369-391.

- (1998) Autonomía colectiva y prevención de riesgos profesionales. Madrid: Ibidem.

QUESADA SEGURA, R. (1997a) "El tratamiento de la autonomía colectiva en la Ley de Prevención de Riesgos Laborales". R. ESCUDERO RODRíGUEZ (coord.) XIV Jornadas Universitarias Andaluzas de Derecho del Trabajo y Relaciones Laborales. La Ley de Prevención de Riesgos Laborales. Sevilla: Consejo Andaluz de Relaciones Laborales, pp. 301-367.

- (1997b) La autonomía colectiva en la Ley de Prevención de Riesgos Laborales. Valencia: Tirant lo Blanch.

Rodríguez HidAlgo, J.G. y H. Álvarez Cuesta (2004) La participación de los trabajadores en el ámbito preventivo. León: Universidad de León.

ROdRíGUEZ-SAÑUdo GUTIÉRREZ, F. (1979) "Participación de los trabajadores en la empresa", Revista de Política Social, 121, pp. 415-437.

- (1996) "Representación y participación de los trabajadores en la empresa en materia de prevención". A. OJEDA AVILÉS, M.R. ALARCóN CARACUEL y M.J. RODRÍGUEZ RAMOS (coords.) La prevención de riesgos laborales. Aspectos clave de la Ley 31/1995. Pamplona: Aranzadi, pp. 255-287.

SALA Franco, T. (2007) Derecho de la prevención de riesgos laborales, $3^{\mathrm{a}}$ ed. actualizada por C. BLASCO PELLICER y C. SALCEDo BELTRÁN. Valencia: Tirant lo Blanch.

SAMPER JUAN, J. (2006) "Los sistemas tradicionales y el régimen español de participación de los trabajadores", XVII Congreso Nacional de Derecho del Trabajo y de la Seguridad Social. Gobierno de la empresa y participación de los trabajadores: viejas y nuevas formas institucionales, Salamanca: AEDTSS / Universidad de Salamanca, pp. 195-271.

SEMPERE NAVARRO, A.V. (2008) "Bases para los nuevos derechos de información y consulta en la empresa", Aranzadi Social, 5, pp. 11-22. 
TASCÓN LóPEZ, R. (2008) "La influencia de la dimensión de la empresa en la participación de los trabajadores en la prevención de riesgos laborales". J.J. Fernández Domínguez (dir.) J. FERnándeZ-Costales MUÑIZ (coord.) Prevención y Dimensión de la Empresa. Valladolid: Junta de Castilla y León / Universidad de León, pp. 131-157.

VALDÉS DAL-RÉ, F. (1997) "Los derechos de participación en la Ley de Prevención de Riesgos Laborales". M.E. CASAS BAAMONDE, M.C. PALOMEQUE LÓPEZ y F. VALDÉS DAL-RÉ (coords.) Seguridad y salud en el trabajo. El nuevo derecho de prevención de riesgos profesionales. Madrid: La LeyActualidad, pp. 67-125.

VILLA GIL, L.E. de la (1980) La participación de los trabajadores en la empresa. Madrid: Instituto de Estudios Económicos. 